\title{
Computing knowledge in security protocols under convergent equational theories *
}

\author{
Ştefan Ciobâcă, Stéphanie Delaune, and Steve Kremer \\ LSV, ENS Cachan \& CNRS \& INRIA, France
}

\begin{abstract}
In the symbolic analysis of security protocols, two classical notions of knowledge, deducibility and indistinguishability, yield corresponding decision problems. We propose a procedure for both problems under arbitrary convergent equational theories. Our procedure terminates on a wide range of equational theories. In particular, we obtain a new decidability result for a theory we encountered when studying electronic voting protocols. We also provide a prototype implementation.
\end{abstract}

\section{Introduction}

Cryptographic protocols are small distributed programs that use cryptographic primitives such as encryption and digital signatures to communicate securely over a network. It is essential to gain as much confidence as possible in their correctness. Therefore, symbolic methods have been developed to analyse such protocols $[4,18,20]$. In these approaches, one of the most important aspects is to be able to reason about the knowledge of the attacker.

Traditionally, the knowledge of the attacker is expressed in terms of deducibility (e.g. [20,10]). A message $s$ (intuitively the secret) is said to be deducible from a set of messages $\varphi$ if an attacker is able to compute $s$ from $\varphi$. To perform this computation, the attacker is allowed, for example, to decrypt deducible messages by deducible keys.

However, deducibility is not always sufficient. Consider for example the case where a protocol participant sends over the network the encryption of one of the constants "yes" or "no" (e.g. the value of a vote). Deducibility is not the right notion of knowledge in this case, since both possible values ("yes" and "no") are indeed "known" to the attacker. In this case, a more adequate form of knowledge is indistinguishability (e.g. [1]): is the attacker able to distinguish between two transcripts of the protocol, one running with the value "yes" and the other one running with the value "no"?

In symbolic approaches to cryptographic protocol analysis, the protocol messages and cryptographic primitives (e.g. encryption) are generally modeled using a term algebra. This term algebra is interpreted modulo an equational theory. Using equational theories provides a convenient and flexible framework for modeling cryptographic primitives [15]. For instance, a simple equational theory for symmetric encryption can be specified by the equation $\operatorname{dec}(\operatorname{enc}(x, y), y)=x$. This

\footnotetext{
* This work has been partly supported by the ANR SeSur AVOTÉ.
} 
equation models the fact that decryption cancels out encryption when the same key is used. Different equational theories can also be used to model randomized encryption or even more complex primitives arising when studying electronic voting protocols $[16,5]$ or direct anonymous attestation [6]: blind signatures, trapdoor commitments, zero-knowledge proofs, ...

The two notions of knowledge that we consider do not take into account the dynamic behavior of the protocol. Nevertheless, in order to establish that two dynamic behaviors of a protocol are indistinguishable, an important subproblem is to establish indistinguishability between the sequences of messages generated by the protocol $[20,2]$. Indistinguishability, also called static equivalence in the applied-pi calculus framework [2], plays an important role in the study of guessing attacks (e.g. [13,7]), as well as for anonymity properties in e-voting protocols (e.g. $[16,5])$. This was actually the starting point of this work. During the study of e-voting protocols, we came across several equational theories for which we needed to show static equivalence while no decision procedure for deduction or static equivalence existed.

Our contributions. We provide a procedure for deduction and static equivalence which is correct, in the sense that if it terminates it gives the right answer, for any convergent equational theory. As deduction and static equivalence are undecidable for this class of equational theories [1], the procedure does not always terminate. However, we show that it does terminate for the class of subterm convergent equational theories (already shown decidable in [1]) and several other theories among which the theory of trapdoor commitment encountered in our electronic voting case studies [16].

Our second contribution is an efficient prototype implementation of this generic procedure. Our procedure relies on a simple fixed point computation based on a few saturation rules, making it convenient to implement.

Related work. Many decision procedures have been proposed for deducibility (e.g. $[10,3,17])$ under a variety of equational theories modeling encryption, digital signatures, exclusive OR, and homomorphic operators. Several papers are also devoted to the study of static equivalence. Most of these results introduce a new procedure for each particular theory and even in the case of the general decidability criterion given in $[1,14]$, the algorithm underlying the proof has to be adapted for each particular theory, depending on how the criterion is fulfilled.

The first generic algorithm that has been proposed handles subterm convergent equational theories [1] and covers the classical theories for encryption and signatures. This result is encompassed by the recent work of Baudet et al. [9] in which the authors propose a generic procedure that works for any convergent equational theory, but which may fail or not terminate. This procedure has been implemented in the YAPA tool [8] and has been shown to terminate without failure in several cases (e.g. subterm convergent theories and blind signatures). However, due to its simple representation of deducible terms (represented by a finite set of ground terms), the procedure fails on several interesting equational 
theories like the theory of trapdoor commitments. Our representation of deducible terms overcomes this limitation by including terms with variables which can be substituted by any deducible terms.

Due to a lack of space, the proofs are given in [12].

\section{Formal model}

\subsection{Term algebras}

As usual, messages will be modeled using a term algebra. Let $\mathcal{F}$ be a finite set of function symbols coming with an arity function ar $: \mathcal{F} \rightarrow \mathbb{N}$. Function symbols of arity 0 are called constants. We consider several kind of atoms: an infinite set of names $\mathcal{N}$, an infinite set of variables $\mathcal{X}$ and a set of parameters $\mathcal{P}$. Names are used to represent keys, nonces and other data exchanged during a protocol run, while variables are used as usual. Parameters act as global variables which are used as pointers to messages exchanged during the protocol run. The essential difference between parameters and variables is that parameters can never be safely $\alpha$-renamed.

The set of terms $\mathcal{T}(\mathcal{F}, \mathcal{A})$ built over $\mathcal{F}$ and the atoms in $\mathcal{A}$ is defined as

$$
\begin{aligned}
t, t_{1}, \ldots::= & \text { term } \\
& a
\end{aligned} \quad \begin{aligned}
& \text { atom } a \in \mathcal{A} \\
& f\left(t_{1}, \ldots, t_{k}\right)
\end{aligned} \quad \begin{aligned}
& \text { application of symbol } f \in \mathcal{F}, \operatorname{ar}(f)=k
\end{aligned}
$$

A term $t$ is said to be ground when $t \in \mathcal{T}(\mathcal{F}, \mathcal{N})$. We assume the usual definitions to manipulate terms. We write $\mathrm{fn}(t)$ (resp. $\operatorname{var}(t)$ ) to represent the set of (free) names (resp. variables) that occur in a term $t$ and $\operatorname{st}(t)$ the set of its (syntactic) subterms. This notation is extended to tuples and sets of terms in the usual way. We denote by $|t|$ the size of $t$ defined as the number of symbols that occur in $t$ (variables do not count), and \#T denotes the cardinality of the set $T$.

The set of positions of a term $t$ is written $\operatorname{pos}(t) \subseteq \mathbb{N}^{*}$. If $p$ is a position of $t$ then $\left.t\right|_{p}$ denotes the subterm of $t$ at the position $p$. The term $t[u]_{p}$ is obtained from $t$ by replacing the occurrence of $\left.t\right|_{p}$ at position $p$ with $u$. A context $C$ is a term with ( 1 or more) holes and we write $C\left[t_{1}, \ldots t_{n}\right]$ for the term obtained by replacing these holes with the terms $t_{1}, \ldots, t_{n}$. A context is public if it only consists of function symbols and holes.

Substitutions are written $\sigma=\left\{x_{1} \mapsto t_{1}, \ldots, x_{n} \mapsto t_{n}\right\}$ with $\operatorname{dom}(\sigma)=$ $\left\{x_{1}, \ldots, x_{n}\right\}$. The application of a substitution $\sigma$ to a term $t$ is written $t \sigma$. The substitution $\sigma$ is grounding for $t$ if the resulting term $t \sigma$ is ground. We use the same notations for replacements of names and parameters by terms.

\subsection{Equational theories and rewriting systems}

Equality between terms will generally be interpreted modulo an equational theory. An equational theory $\mathcal{E}$ is defined by a set of equations $M \sim N$ with 
$M, N \in \mathcal{T}(\mathcal{F}, \mathcal{X})$. Equality modulo $\mathcal{E}$, written $=\mathcal{E}$, is defined to be the smallest equivalence relation on terms such that $M={ }_{\mathcal{E}} N$ for all $M \sim N \in \mathcal{E}$ and which is closed under substitution of terms for variables and application of contexts.

It is often more convenient to manipulate rewriting systems than equational theories. A rewriting system $\mathcal{R}$ is a set of rewriting rules $l \rightarrow r$ where $l, r \in \mathcal{T}(\mathcal{F}, \mathcal{X})$ and $\operatorname{var}(r) \subseteq \operatorname{var}(l)$. A term $t$ rewrites to $t^{\prime}$ by $\mathcal{R}$, denoted by $t \rightarrow_{\mathcal{R}} t^{\prime}$, if there exists $l \rightarrow r \in \mathcal{R}$, a position $p \in \operatorname{pos}(t)$ and a substitution $\sigma$ such that $\left.t\right|_{p}=l \sigma$ and $t^{\prime}=t[r \sigma]_{p}$. We denote by $\rightarrow_{\mathcal{R}}^{+}$the transitive closure of $\rightarrow_{\mathcal{R}}, \rightarrow_{\mathcal{R}}^{*}$ its reflexive and transitive closure, and $=_{\mathcal{R}}$ its reflexive, symmetric and transitive closure.

A rewrite system $\mathcal{R}$ is convergent if it is terminating, i.e. there is no infinite chains $u_{1} \rightarrow_{\mathcal{R}} u_{2} \rightarrow_{\mathcal{R}} \ldots$, and confluent, i.e. for every term $u$ such that $u \rightarrow_{\mathcal{R}}^{*} u_{1}$ and $u \rightarrow_{\mathcal{R}}^{*} u_{2}$, there exists $v$ such that $u_{1} \rightarrow_{\mathcal{R}}^{*} v$ and $u_{2} \rightarrow_{\mathcal{R}}^{*} v$. A term $u$ is in $\mathcal{R}$-normal form if there is no term $u^{\prime}$ such that $u \rightarrow_{\mathcal{R}} u^{\prime}$. If $u \rightarrow_{\mathcal{R}}^{*} u^{\prime}$ and $u^{\prime}$ is in $\mathcal{R}$-normal form then $u^{\prime}$ is an $\mathcal{R}$-normal form of $u$. When this reduced form is unique (in particular if $\mathcal{R}$ is convergent), we write $u^{\prime}=u \downarrow_{\mathcal{R}_{\mathcal{E}}}$.

We are particularly interested in theories $\mathcal{E}$ that can be represented by a convergent rewrite system $\mathcal{R}$, i.e. theories for which there exists a convergent rewrite system $\mathcal{R}$ such that the two relations $=_{\mathcal{R}}$ and $=_{\mathcal{E}}$ coincide. Given an equational theory $\mathcal{E}$ we define the corresponding rewriting system $\mathcal{R}_{\mathcal{E}}$ by orienting all equations in $\mathcal{E}$ from left to right, i.e., $\mathcal{R}_{\mathcal{E}}=\{l \rightarrow r \mid l \sim r \in \mathcal{E}\}$. We say that $\mathcal{E}$ is convergent if $\mathcal{R}_{\mathcal{E}}$ is convergent.

Example 1. A classical equational theory modelling symmetric encryption is $\mathcal{E}_{\text {enc }}=\{\operatorname{dec}(\operatorname{enc}(x, y), y) \sim x\}$.

As a running example we consider a slight extension of this theory modelling malleable encryption

$$
\mathcal{E}_{\text {mal }}=\mathcal{E}_{\text {enc }} \cup\{\operatorname{mal}(\operatorname{enc}(x, y), z) \sim \operatorname{enc}(z, y)\} .
$$

This malleable encryption scheme allows one to arbitrarily change the plaintext of an encryption. This theory certainly does not model a realistic encryption scheme but it yields a simple example of a theory which illustrates well our procedures. In particular all existing decision procedure we are aware of fail on this example. The rewriting system $\mathcal{R}_{\mathcal{E}_{\text {mal }}}$ is convergent.

From now on, we assume given a convergent equational theory $\mathcal{E}$ built over a signature $\mathcal{F}$ and represented by the convergent rewriting system $\mathcal{R}_{\mathcal{E}}$.

\subsection{Deducibility and static equivalence}

In order to describe the messages observed by an attacker, we consider the following notion of frame that comes from the applied-pi calculus [2].

A frame $\varphi$ is a sequence of messages $u_{1}, \ldots, u_{n}$ meaning that the attacker observed each of these messages in the given order. Furthermore, we distinguish the names that the attacker knows from those that were freshly generated by others and that are a priori unknown by the attacker. Formally, a frame is defined as $\nu \tilde{n} . \sigma$ where $\tilde{n}$ is its set of bound names, denoted by $\operatorname{bn}(\varphi)$, and a 
replacement $\sigma=\left\{w_{1} \mapsto u_{1}, \ldots, w_{n} \mapsto u_{n}\right\}$. The parameters $w_{1}, \ldots, w_{n}$ enable us to refer to $u_{1}, \ldots, u_{n} \in \mathcal{T}(\mathcal{F}, \mathcal{N})$. The domain $\operatorname{dom}(\varphi)$ of $\varphi$ is $\left\{w_{1}, \ldots, w_{n}\right\}$.

Given terms $M$ and $N$ such that $\operatorname{fn}(M, N) \cap \tilde{n}=\emptyset$, we sometimes write $(M=\mathcal{E} N) \varphi($ resp. $M \varphi)$ instead of $M \sigma={ }_{\mathcal{E}} N \sigma($ resp. $M \sigma)$.

Definition 1 (deducibility). Let $\varphi$ be a frame. A ground term $t$ is deducible in $\mathcal{E}$ from $\varphi$, written $\varphi \vdash_{\mathcal{E}} t$, if there exists $M \in \mathcal{T}(\mathcal{F}, \mathcal{N} \cup \operatorname{dom}(\varphi))$, called the recipe, such that $\operatorname{fn}(M) \cap \operatorname{bn}(\varphi)=\emptyset$ and $M \varphi={ }_{\mathcal{E}} t$.

Deducibility does not always suffice for expressing the knowledge of an attacker. For instance deducibility does not allow one to express indistinguishability between two sequences of messages. This is important when defining the confidentiality of a vote or anonymity-like properties. This motivates the following notion of static equivalence introduced in [2].

Definition 2 (static equivalence). Let $\varphi_{1}$ and $\varphi_{2}$ be two frames such that $\operatorname{bn}\left(\varphi_{1}\right)=\operatorname{bn}\left(\varphi_{2}\right)$. They are statically equivalent in $\mathcal{E}$, written $\varphi_{1} \approx_{\mathcal{E}} \varphi_{2}$, if

$-\operatorname{dom}\left(\varphi_{1}\right)=\operatorname{dom}\left(\varphi_{2}\right)$

- for all terms $M, N \in \mathcal{T}\left(\mathcal{F}, \mathcal{N} \cup \operatorname{dom}\left(\varphi_{1}\right)\right)$ such that $\operatorname{fn}(M, N) \cap \operatorname{bn}\left(\varphi_{1}\right)=\emptyset$

$$
(M=\mathcal{E} N) \varphi_{1} \Leftrightarrow\left(M={ }_{\mathcal{E}} N\right) \varphi_{2} .
$$

Example 2. Consider the two frames described below:

$$
\varphi_{1}=\nu a, k .\left\{w_{1} \mapsto e n c(a, k)\right\} \quad \text { and } \quad \varphi_{2}=\nu a, k .\left\{w_{1} \mapsto e n c(b, k)\right\}
$$

We have that $b$ and enc $(c, k)$ are deducible from $\varphi_{2}$ in $\mathcal{E}_{m a l}$ with recipes $b$ and $\operatorname{mal}\left(w_{1}, c\right)$ respectively. We have that $\varphi_{1} \not \mathcal{E}_{\mathcal{E}_{\text {mal }}} \varphi_{2}$ since $\left(w_{1} \neq \mathcal{E}_{\text {mal }} \operatorname{mal}\left(w_{1}, b\right)\right) \varphi_{1}$ while $\left(w_{1}=\mathcal{E}_{\text {mal }} \operatorname{mal}\left(w_{1}, b\right)\right) \varphi_{2}$. Note that $\varphi_{1} \approx_{\mathcal{E}_{\text {enc }}} \varphi_{2}$ (in the theory $\mathcal{E}_{\text {enc }}$ ).

\section{Procedures for deduction and static equivalence}

In this section we describe our procedures for checking deducibility and static equivalence on convergent equational theories. After some preliminary definitions, we present the main part of our procedure, i.e. a set of saturation rules used to reach a fixed point. Then, we show how to use this saturation procedure to decide deducibility and static equivalence. Soundness and completeness of the saturation procedure are stated in Theorem 1 and detailed in Section 4.

Since both problems are undecidable for arbitrary convergent equational theories [1], our saturation procedure does not always terminate. In Section 5, we exhibit (classes of) equational theories for which the saturation terminates.

\subsection{Preliminary definitions}

The main objects that will be manipulated by our procedure are facts, which are either deduction facts or equational facts. 
Definition 3 (facts). A deduction fact (resp. an equational fact) is an expression denoted $\left[U \triangleright u \mid\left\{X_{1} \triangleright t_{1}, \ldots, X_{n} \triangleright t_{n}\right\}\right]$ (resp. $\left[U \sim V \mid\left\{X_{1} \triangleright t_{1}, \ldots, X_{n} \triangleright\right.\right.$ $\left.t_{n}\right\}$ ]) where $X_{i} \triangleright t_{i}(1 \leq i \leq n)$ are called the side conditions of the fact. Moreover, we assume that:

$$
\begin{aligned}
& -u, t_{1}, \ldots, t_{n} \in \mathcal{T}(\mathcal{F}, \mathcal{N} \cup \mathcal{X}) \text { with } \operatorname{var}(u) \subseteq \operatorname{var}\left(t_{1}, \ldots, t_{n}\right) \\
& -U, V \in \mathcal{T}(\mathcal{F}, \mathcal{N} \cup \mathcal{X} \cup \mathcal{P}) \text { and } X_{1}, \ldots, X_{n} \in \mathcal{X} \\
& -\operatorname{var}\left(U, V, X_{1}, \ldots, X_{n}\right) \cap \operatorname{var}\left(u, t_{1}, \ldots, t_{n}\right)=\emptyset
\end{aligned}
$$

We say that a fact is solved if $t_{i} \in \mathcal{X}(1 \leq i \leq k)$. Otherwise, it is unsolved. $A$ deduction fact is well-formed if it is unsolved or if $u \notin \mathcal{X}$.

A fact makes a statement about a frame. We read $\left[U \triangleright u \mid\left\{X_{1} \triangleright t_{1}, \ldots, X_{n} \triangleright\right.\right.$ $\left.t_{n}\right\}$ ] (resp. $\left[U \sim V \mid\left\{X_{1} \triangleright t_{1}, \ldots, X_{n} \triangleright t_{n}\right\}\right]$ ) as " $u$ is deducible with recipe $U$ (resp. $U$ is equal to $V$ ) if $t_{i}$ is deducible with recipe $X_{i}$ (for all $1 \leq i \leq n$ )". For notational convenience we sometimes omit curly braces for the set of side conditions and write $\left[U \triangleright u \mid X_{1} \triangleright t_{1}, \ldots, X_{n} \triangleright t_{n}\right]$. When $n=0$ we simply write $[U \triangleright u]$ or $[U \sim V]$.

We say that two facts are equivalent if they are equal up to bijective renaming of variables. In the following we implicitly suppose that all operations are carried out modulo the equivalence classes. In particular set union will not add equivalent facts and inclusion will test for equivalent facts. Also, we allow on-the-fly renaming of variables in facts to avoid variable clashes.

We now introduce the notion of generation of a term $t$ from a set of facts $\mathrm{F}$. Intuitively, we say that a term $t$ is generated if it can be syntactically "deduced" from $\mathrm{F}$.

Definition 4 (generation). Let $\mathrm{F}$ be a finite set of well-formed deduction facts. $A$ term $t$ is generated by $\mathrm{F}$ with recipe $R$, written $\mathrm{F} \vdash^{R} t$, if

1. either $t=x \in \mathcal{X}$ and $R=x$;

2. or there exist a solved fact $\left[R_{0} \triangleright t_{0} \mid X_{1} \triangleright x_{1}, \ldots, X_{n} \triangleright x_{n}\right] \in \mathrm{F}$, some terms $R_{i}$ for $1 \leq i \leq n$ and a substitution $\sigma$ with $\operatorname{dom}(\sigma) \subseteq \operatorname{var}\left(t_{0}\right)$ such that $t=t_{0} \sigma$, $R=R_{0}\left[X_{1} \mapsto R_{1}, \ldots, X_{k} \mapsto R_{k}\right]$, and $\mathrm{F} \vdash{ }^{R_{i}} x_{i} \sigma$ for every $1 \leq i \leq n$.

A term $t$ is generated by $\mathrm{F}$, written $\mathrm{F} \vdash t$, if there exists $R$ such that $\mathrm{F} \vdash^{R} t$.

From this definition follows a simple recursive algorithm for effectively deciding whether $\mathrm{F} \vdash t$, providing also the recipe. Termination is ensured by the fact that $\left|x_{i} \sigma\right|<|t|$ for every $1 \leq i \leq n$. Note that using memoization we can obtain an algorithm in polynomial time.

Given a finite set of equational facts $\mathrm{E}$ and terms $M, N$, we write $\mathrm{E} \models M \sim N$ if $M \sim N$ is a consequence, in the usual first order theory of equality, of

$$
\left\{U \sigma \sim V \sigma \mid\left[U \sim V \mid X_{1} \triangleright x_{1}, \ldots, X_{k} \triangleright x_{k}\right] \in \mathrm{E}\right\} \text { where } \sigma=\left\{X_{i} \mapsto x_{i}\right\}_{1 \leq i \leq k} .
$$

Note that it may be the case that $x_{i}=x_{j}$ for $i \neq j$ (whereas $X_{i} \neq X_{j}$ ). 


\subsection{Saturation procedure}

We define for each fact its canonical form which is obtained by first applying rule (1) and then rule (2) defined below. The idea is to ensure that each variable $x_{i}$ occurs at most once in the side conditions and to get rid of those variables that do not occur in $t$. Unsolved deduction facts are kept unchanged.

(1) $\frac{\left[R \triangleright t \mid X_{1} \triangleright x_{1}, \ldots, X_{k} \triangleright x_{k}\right]\{i, j\} \subseteq\{1, \ldots, n\} j \neq i \text { and } x_{j}=x_{i}}{\left[R\left[X_{i} \mapsto X_{j}\right] \triangleright t \mid X_{1} \triangleright x_{1}, \ldots, X_{i-1} \triangleright x_{i-1}, X_{i+1} \triangleright x_{i+1}, \ldots, X_{k} \triangleright x_{k}\right]}$

(2) $\frac{\left[R \triangleright t \mid X_{1} \triangleright x_{1}, \ldots, X_{k} \triangleright x_{k}\right] \quad x_{i} \notin \operatorname{var}(t)}{\left[R \triangleright t \mid X_{1} \triangleright x_{1}, \ldots, X_{i-1} \triangleright x_{i-1}, X_{i+1} \triangleright x_{i+1}, \ldots, X_{k} \triangleright x_{k}\right]}$

A knowledge base is a tuple $(\mathrm{F}, \mathrm{E})$ where $\mathrm{F}$ is a finite set of well-formed deduction facts that are in canonical form and $E$ a finite set of equational facts.

Definition 5 (update). Given a fact $\mathrm{f}=\left[R \triangleright t \mid X_{1} \triangleright t_{1}, \ldots, X_{n} \triangleright t_{n}\right]$ and a knowledge base $(\mathrm{F}, \mathrm{E})$, the update of $(\mathrm{F}, \mathrm{E})$ by $\mathrm{f}$, written $(\mathrm{F}, \mathrm{E}) \oplus \mathrm{f}$, is defined as

$$
\left\{\begin{array}{c}
\left(\mathrm{F} \cup\left\{\mathrm{f}^{\prime}\right\}, \mathrm{E}\right) \\
\text { where } \mathrm{f}^{\prime} \text { is the canonical form of } \mathrm{f} \\
\left(\mathrm{F}, \mathrm{E} \cup\left\{\left[R^{\prime} \sim R\left\{X_{i} \mapsto t_{i}\right\}_{1 \leq i \leq n}\right\}\right]\right) \\
\text { where } \mathrm{F} \vdash^{\prime} R^{\prime} t
\end{array} \quad \begin{array}{ll}
\text { if } \mathrm{f} \text { is solved and } \mathrm{F} \vdash t \text { useless fact } \\
(\mathrm{F} \cup\{\mathrm{f}\}, \mathrm{E}) & \text { if } \mathrm{f} \text { is not solved } \quad \text { unsolved fact }
\end{array}\right.
$$

The choice of the recipe $R^{\prime}$ in the useless fact case is defined by the implementation. While this choice does not influence the correctness of the procedure, it might influence its termination as we will see later. Note that, the result of updating a knowledge base by a (possibly not well-formed and/or not canonical) fact is again a knowledge base. Facts that are not well-formed will be captured by the useless fact case, which adds an equational fact.

Initialisation. Given a frame $\varphi=\nu \tilde{n} .\left\{w_{1} \mapsto t_{1}, \ldots, w_{n} \mapsto t_{n}\right\}$, our procedure starts from an initial knowledge base associated to $\varphi$ and defined as follows:

$$
\begin{aligned}
& \operatorname{Init}(\varphi)=\quad(\emptyset, \emptyset) \\
& \bigoplus_{1 \leq i \leq n} \quad\left[w_{i} \triangleright t_{i}\right] \\
& \bigoplus_{n \in \mathrm{fn}(\varphi)}[n \triangleright n] \\
& \bigoplus_{f \in \mathcal{F}}\left[f\left(X_{1}, \ldots, X_{k}\right) \triangleright f\left(x_{1}, \ldots, x_{k}\right) \mid X_{1} \triangleright x_{1}, \ldots \triangleright X_{k} \triangleright x_{k}\right]
\end{aligned}
$$

Example 3. Consider the rewriting system $\mathcal{R}_{\mathcal{E}_{\text {mal }}}$ and $\varphi_{2}=\nu a, k .\left\{w_{1} \mapsto e n c(b, k)\right\}$. The knowledge base Init $\left(\varphi_{2}\right)$ is made up of the following deduction facts:

$$
\begin{aligned}
& {\left[w_{1} \triangleright \operatorname{enc}(b, k) \mid \emptyset\right] \quad\left(\mathrm{f}_{1}\right) \quad\left[\operatorname{enc}\left(Y_{1}, Y_{2}\right) \triangleright \operatorname{enc}\left(y_{1}, y_{2}\right) \mid Y_{1} \triangleright y_{1}, Y_{2} \triangleright y_{2}\right] \quad\left(\mathrm{f}_{3}\right)} \\
& {\left[\begin{array}{llllllll}
b & \triangleright & b & \emptyset
\end{array} \quad\left(\mathrm{f}_{2}\right) \quad\left[\operatorname{dec}\left(Y_{1}, Y_{2}\right) \triangleright \operatorname{dec}\left(y_{1}, y_{2}\right) \mid Y_{1} \triangleright y_{1}, Y_{2} \triangleright y_{2}\right] \quad\left(\mathrm{f}_{4}\right)\right.} \\
& {\left[\operatorname{mal}\left(Y_{1}, Y_{2}\right) \triangleright \operatorname{mal}\left(y_{1}, y_{2}\right) \mid Y_{1} \triangleright y_{1}, Y_{2} \triangleright y_{2}\right] \quad\left(\mathrm{f}_{5}\right)}
\end{aligned}
$$


Saturation. The main part of our procedure consists in saturating the knowledge base Init $(\varphi)$ by means of the transformation rules described in Figure 1. The rule Narrowing is designed to apply a rewriting step on an existing deduction fact. Intuitively, this rule allows us to get rid of the equational theory and nevertheless ensure that the generation of deducible terms is complete. The rule F-Solving is used to instantiate an unsolved side condition of an existing deduction fact. Unifying and E-Solving add equational facts which remember when different recipes for the same term exist.

Note that this procedure may not terminate and that the fixed point may not be unique.

We write $\Longrightarrow^{*}$ for the reflexive and transitive closure of $\Longrightarrow$.

\section{Narrowing}

$\mathrm{f}=\left[M \triangleright C[t] \mid X_{1} \triangleright x_{1}, \ldots, X_{k} \triangleright x_{k}\right] \in \mathrm{F}, l \rightarrow r \in \mathcal{R}_{\mathcal{E}}$

with $t \notin \mathcal{X}, \sigma=\operatorname{mgu}(l, t)$ and $\operatorname{var}(\mathbf{f}) \cap \operatorname{var}(l)=\emptyset$.

$$
(\mathrm{F}, \mathrm{E}) \Longrightarrow(\mathrm{F}, \mathrm{E}) \oplus \mathrm{f}_{0}
$$

where $\mathrm{f}_{0}=\left[M \triangleright(C[r]) \sigma \mid X_{1} \triangleright x_{1} \sigma, \ldots, X_{k} \triangleright x_{k} \sigma\right]$.

\section{F-Solving}

$\mathrm{f}_{1}=\left[M \triangleright t \mid X_{0} \triangleright t_{0}, \ldots, X_{k} \triangleright t_{k}\right], \mathrm{f}_{2}=\left[N \triangleright s \mid Y_{1} \triangleright y_{1}, \ldots, Y_{\ell} \triangleright y_{\ell}\right] \in \mathrm{F}$

with $t_{0} \notin \mathcal{X}, \quad \sigma=\operatorname{mgu}\left(s, t_{0}\right)$ and $\operatorname{var}\left(\mathrm{f}_{1}\right) \cap \operatorname{var}\left(\mathrm{f}_{2}\right)=\emptyset$.

$$
(\mathrm{F}, \mathrm{E}) \Longrightarrow(\mathrm{F}, \mathrm{E}) \oplus \mathrm{f}_{0}
$$

where $\mathrm{f}_{0}=\left[M\left\{X_{0} \mapsto N\right\} \triangleright t \sigma \mid X_{1} \triangleright t_{1} \sigma, \ldots, X_{k} \triangleright t_{k} \sigma, Y_{1} \triangleright y_{1} \sigma, \ldots, Y_{\ell} \triangleright y_{\ell} \sigma\right]$.

\section{Unifying}

$\mathrm{f}_{1}=\left[M \triangleright t \mid X_{1} \triangleright x_{1}, \ldots, X_{k} \triangleright x_{k}\right], \mathrm{f}_{2}=\left[N \triangleright s \mid Y_{1} \triangleright y_{1}, \ldots, Y_{\ell} \triangleright y_{\ell}\right] \in \mathrm{F}$

with $\sigma=\operatorname{mgu}(s, t)$ and $\operatorname{var}\left(\mathrm{f}_{1}\right) \cap \operatorname{var}\left(\mathrm{f}_{2}\right)=\emptyset$.

$$
(\mathrm{F}, \mathrm{E}) \Longrightarrow\left(\mathrm{F}, \mathrm{E} \cup\left\{\mathrm{f}_{0}\right\}\right)
$$

where $\mathrm{f}_{0}=\left[M \sim N \mid\left\{X_{i} \triangleright x_{i} \sigma\right\}_{1 \leq i \leq k} \cup\left\{Y_{i} \triangleright y_{i} \sigma\right\}_{1 \leq i \leq \ell}\right]$.

\section{E-Solving}

$\mathrm{f}_{1}=\left[U \sim V \mid Y \triangleright s, X_{1} \triangleright t_{1}, \ldots, X_{k} \triangleright t_{k}\right] \in \mathrm{E}, \mathrm{f}_{2}=\left[M \triangleright t \mid Y_{1} \triangleright y_{1}, \ldots, Y_{\ell} \triangleright y_{\ell}\right\} \in \mathrm{F}$ with $s \notin \mathcal{X}, \sigma=\operatorname{mgu}(s, t)$ and $\operatorname{var}\left(\mathrm{f}_{1}\right) \cap \operatorname{var}\left(\mathrm{f}_{2}\right)=\emptyset$.

$(\mathrm{F}, \mathrm{E}) \Longrightarrow\left(\mathrm{F}, \mathrm{E} \cup\left\{\mathrm{f}_{0}\right\}\right)$
where $\mathrm{f}_{0}=\left[U\{Y \mapsto M\} \sim V\{Y \mapsto M\} \mid\left\{X_{i} \triangleright t_{i} \sigma\right\}_{1 \leq i \leq k} \cup\left\{Y_{i} \triangleright y_{i} \sigma\right\}_{1 \leq i \leq \ell}\right]$.

Fig. 1. Saturation rules

Example 4. Continuing Example 3, we illustrate the saturation procedure. We can apply the rule Narrowing on $\mathrm{f}_{4}$ and on the rewrite rule $\operatorname{dec}(\operatorname{enc}(x, y), y) \rightarrow x$, as well as on $\mathrm{f}_{5}$ and the rewrite rule $\operatorname{mal}(\operatorname{enc}(x, y), z) \rightarrow \operatorname{enc}(z, y)$, thereby adding 
the facts

$$
\begin{array}{cc|c}
{\left[\operatorname{dec}\left(Y_{1}, Y_{2}\right) \triangleright\right.} & x & \left.Y_{1} \triangleright \operatorname{enc}(x, y), Y_{2} \triangleright y\right] \\
{\left[\operatorname{mal}\left(Y_{1}, Y_{2}\right) \triangleright \operatorname{enc}(z, y)\right.} & \left.\mid Y_{1} \triangleright \operatorname{enc}(x, y), Y_{2} \triangleright z\right]
\end{array}
$$

The facts $f_{6}$ and $f_{7}$ are not solved and we can apply the rule F-Solving with $f_{1}$, thereby adding the facts:

$$
\left[\operatorname{dec}\left(w_{1}, Y_{2}\right) \triangleright b \mid Y_{2} \triangleright k\right]\left(\mathrm{f}_{8}\right) \quad\left[\operatorname{mal}\left(w_{1}, Y_{2}\right) \triangleright \operatorname{enc}(z, k) \mid Y_{2} \triangleright z\right]\left(\mathbf{f}_{9}\right)
$$

Rule Unifying can be used on facts $\mathrm{f}_{1} / \mathrm{f}_{3}, \mathrm{f}_{3} / \mathrm{f}_{9}$ as well as $\mathrm{f}_{1} / \mathrm{f}_{9}$ to add equational facts. This third case allows one to obtain $\mathrm{f}_{10}=\left[w_{1} \sim \operatorname{mal}\left(w_{1}, Y_{2}\right) \mid Y_{2} \triangleright b\right]$ which can be solved (using E-Solving with $\mathrm{f}_{2}$ ) to obtain $\mathrm{f}_{11}=\left[w_{1} \sim \operatorname{mal}\left(w_{1}, b\right)\right]$. Because of lack of space we do not detail the remaining rule applications. When reaching a fixed point the knowledge base contains the solved facts $f_{9}$ and $f_{11}$ as well as those in $\operatorname{Init}\left(\varphi_{2}\right)$.

We now state the soundness and completeness of our transformation rules. The technical lemmas used to prove this result are detailed in Section 4.

Theorem 1 (soundness and completeness). Let $\varphi$ be a frame and (F, E) be a saturated knowledge base such that $\operatorname{Init}(\varphi) \Longrightarrow^{*}(\mathrm{~F}, \mathrm{E})$. Let $t \in \mathcal{T}(\mathcal{F}, \mathcal{N})$ and

$\mathrm{F}^{+}=\mathrm{F} \cup\{[n \triangleright n] \mid n \in \mathrm{fn}(t) \backslash \mathrm{bn}(\varphi)\}$. We have that:

1. For all $M \in \mathcal{T}(\mathcal{F}, \mathcal{N} \cup \operatorname{dom}(\varphi))$ such that $\operatorname{fn}(M) \cap \operatorname{bn}(\varphi)=\emptyset$, we have that

$$
M \varphi={ }_{\mathcal{E}} t \Leftrightarrow \exists N, \mathrm{E} \models M \sim N \text { and } \mathrm{F}^{+} \vdash^{N} t \downarrow_{\mathcal{R}_{\mathcal{E}}}
$$

2. For all $M, N \in \mathcal{T}(\mathcal{F}, \mathcal{N} \cup \operatorname{dom}(\varphi))$ such that $\operatorname{fn}(M, N) \cap \operatorname{bn}(\varphi)=\emptyset$, we have

$$
\left(M={ }_{\mathcal{E}} N\right) \varphi \Leftrightarrow \mathrm{E} \models M \sim N .
$$

\subsection{Application to deduction and static equivalence}

Procedure for deduction. Let $\varphi$ be a frame and $t$ be a ground term. The procedure for checking $\varphi \vdash_{\mathcal{E}} t$ is described bellow. Its correctness is a direct consequence of Theorem 1, Item 1 .

1. Apply the saturation rules to obtain (if any) a saturated knowledge base $(\mathrm{F}, \mathrm{E}) \operatorname{such}$ that $\operatorname{Init}(\varphi) \Longrightarrow^{*}(\mathrm{~F}, \mathrm{E})$. Let $^{+}=\mathrm{F} \cup\{[n \triangleright n] \mid n \in \mathrm{fn}(t) \backslash \operatorname{bn}(\varphi)\}$.

2. Return yes if there exists $N$ such that $\mathrm{F}^{+} \vdash^{N} t_{\downarrow_{\mathcal{R}_{\mathcal{E}}}}$ (that is, the $\mathcal{R}_{\mathcal{E}}$-normal form of $t$ is generated by $\mathrm{F}$ with recipe $N$ ); otherwise return no.

Example 5. We continue our running example. Let (F,E) be the knowledge base obtained from Init $\left(\varphi_{2}\right)$ described in Example 4. We show that $\varphi_{2} \vdash$ enc $(c, k)$ and $\varphi_{2} \vdash b$. Indeed we have that $\mathrm{F} \cup\{[c \triangleright c]\} \vdash^{\operatorname{mal}\left(w_{1}, c\right)}$ enc $(c, k)$ using facts $\mathrm{f}_{9}$ and $[c \triangleright c]$, and $\mathrm{F} \vdash^{b} b$ using fact $\mathrm{f}_{2}$. 
Procedure for static equivalence. Let $\varphi_{1}$ and $\varphi_{2}$ be two frames. The procedure for checking $\varphi_{1} \approx_{\mathcal{E}} \varphi_{2}$ runs as follows:

1. Apply the transformation rules to obtain (if possible) two saturated knowledge bases $\left(\mathrm{F}_{i}, \mathrm{E}_{i}\right), i=1,2$ such that $\operatorname{Init}\left(\varphi_{i}\right) \Longrightarrow^{*}\left(\mathrm{~F}_{i}, \mathrm{E}_{i}\right), i=1,2$.

2. For $\{i, j\}=\{1,2\}$, for every solved fact $\left[M \sim N \mid X_{1} \triangleright x_{1}, \ldots, X_{k} \triangleright x_{k}\right]$ in $\mathrm{E}_{i}$, check if $\left(M\left\{X_{1} \mapsto x_{1}, \ldots, X_{k} \mapsto x_{k}\right\}=\mathcal{E} N\left\{X_{1} \mapsto x_{1}, \ldots, X_{k} \mapsto x_{k}\right\}\right) \varphi_{j}$.

3 . If so return yes; otherwise return no.

Proof. (Sketch) If the algorithm returns no, then there exists an equation that holds in one frame but not in the other; therefore, the two frames are not statically equivalent.

Assume that the algorithm returns yes. Let $M \sim N$ be an arbitrary equation that holds in $\varphi_{1}$. By Theorem 1, Item 2, we have that $\mathrm{E}_{1}=M \sim N$. As all equations in $\mathrm{E}_{1}$ also hold in $\varphi_{2}$, and because $\mathrm{E}_{1} \models M \sim N$, it follows that $M \sim N$ holds in $\varphi_{2}$. We have shown that all equations that hold in $\varphi_{1}$ also hold in $\varphi_{2}$. Similarly, all equations that hold in $\varphi_{2}$ hold in $\varphi_{1}$ and therefore the two frames are statically equivalent.

Example 6. Consider again the frames $\varphi_{1}$ and $\varphi_{2}$ which are not statically equivalent (see Example 2). Our procedure answers no since $\left[\operatorname{mal}\left(w_{1}, b\right) \sim w_{1}\right] \in \mathrm{E}_{2}$ whereas $\left(\operatorname{mal}\left(w_{1}, b\right) \neq \mathcal{E}_{\text {mal }} w_{1}\right) \varphi_{1}$.

\section{Soundness and completeness}

In this section we give the key results to prove Theorem 1 . The soundness of our saturation procedure relies on Lemma 1 whereas its completeness is more involved: the key propositions are stated below.

Intuitively Lemma 1 states that any ground term which can be generated is indeed deducible. Similarly all equations which are consequences of the knowledge base are true equations in the initial frame. The soundness of our saturation procedure can be easily derived from this lemma.

Lemma 1 (soundness). Let $\varphi$ be a frame and (F,E) be a knowledge base such that $\operatorname{Init}(\varphi) \Longrightarrow{ }^{*}(\mathrm{~F}, \mathrm{E})$. Let $t \in \mathcal{T}(\mathcal{F}, \mathcal{N}), M, N \in \mathcal{T}(\mathcal{F}, \mathcal{N} \cup \operatorname{dom}(\varphi))$ such that $\mathrm{fn}(M, N) \cap \operatorname{bn}(\varphi)=\emptyset$, and $\mathrm{F}^{+}=\mathrm{F} \cup\{[n \triangleright n] \mid n \in \mathrm{fn}(t) \backslash \mathrm{bn}(\varphi)\}$. We have that:

1. $\mathrm{F}^{+} \vdash^{M} t \Rightarrow M \varphi=\mathcal{E} t$; and

2. $\mathrm{E} \models M \sim N \Rightarrow(M=\mathcal{E} N) \varphi$.

We now give two propositions that are used to show the completeness of the saturation rules. The first one states that whenever there exist two recipes to generate a ground term from $F$ then the equation on the two recipes is a consequence of $E$.

Proposition 1 (completeness, equation). Let (F,E) be a saturated knowledge base, and $M, N$ be two terms such that $\mathrm{F} \vdash^{M} t$ and $\mathrm{F} \vdash^{N} t$ for some ground term $t$. Then, we have that $\mathrm{E}=M \sim N$. 
Next we show that whenever a ground term (not necessarily in normal form) can be generated then its normal form can also be generated and there exists an equation on the two recipes.

Proposition 2 (completeness, reduction). Let (F,E) be a saturated knowledge base, $M$ a term and $t$ a ground term such that $\mathrm{F} \vdash^{M} t$ and $t \downarrow_{\mathcal{R}_{\mathcal{E}}} \neq t$. Then there exists $M^{\prime}$ and $t^{\prime}$ such that $\mathrm{F} \vdash^{M^{\prime}} t^{\prime}$ with $t \rightarrow_{\mathcal{R}_{\mathcal{E}}}^{+} t^{\prime}$ and $\mathrm{E}=M \sim M^{\prime}$.

Relying on these propositions, we can show completeness of our saturation procedure (i.e. $\Rightarrow$ of Theorem 1 ).

1. To prove Item 1 , we first observe that if $t$ is deducible from $\varphi$ modulo $\mathcal{E}$ then $\mathrm{F}^{+} \vdash^{M^{\prime}} t_{0}$ for some $M^{\prime}$ and $t_{0}$ such that $\mathrm{E} \models M \sim M^{\prime}$ and $t_{0} \rightarrow^{*} t \downarrow_{\mathcal{R}_{\mathcal{E}}}$. Actually $M^{\prime}$ differs from $M$ by the fact that some public names that do not occur in the knowledge base are replaced by fresh variables. Then, we rely on Proposition 2 and we show the result by induction on $t_{0}$ equipped with the order $<$ induced by the rewriting relation $\left(t<t^{\prime}\right.$ iff $\left.t \rightarrow^{+} t^{\prime}\right)$.

2. Now, to prove Item 2, we apply the result shown in item 1 on $M \varphi={ }_{\mathcal{E}} t$ and $N \varphi={ }_{\mathcal{E}} t$ where $t=M \varphi \downarrow_{\mathcal{R}_{\mathcal{E}}}=N \varphi \downarrow_{\mathcal{R}_{\mathcal{E}}}$. We deduce that there exist $M^{\prime}$ and $N^{\prime}$ such that $\mathrm{E} \models M \sim M^{\prime}, \mathrm{F}^{+} \vdash^{M^{\prime}} t, \mathrm{E} \mid=N \sim N^{\prime}$, and $\mathrm{F}^{+} \vdash^{N^{\prime}} t$. Then, Proposition 1 allows one to deduce that $\mathrm{E} \models M^{\prime} \sim N^{\prime}$, thus $\mathrm{E} \models M \sim N$.

\section{Termination}

As already announced the saturation process will not always terminate.

Example 7. Consider the convergent rewriting system consisting of the single rule $f(g(x)) \rightarrow g(h(x))$ and the frame $\phi=\nu a .\left\{w_{1} \mapsto g(a)\right\}$. We have that

$$
\operatorname{Init}(\varphi) \supseteq\left\{\left[w_{1} \triangleright g(a)\right],[f(X) \triangleright f(x) \mid X \triangleright x]\right\} .
$$

By Narrowing we can add the fact $\mathrm{f}_{1}=[f(X) \triangleright g(h(x)) \mid X \triangleright g(x)]$. Then we can apply F-Solving to solve its side condition $X \triangleright g(x)$ with the fact $\left[w_{1} \triangleright g(a)\right]$ yielding the solved fact $\left[f\left(w_{1}\right) \triangleright g(h(a))\right]$. Now, applying iteratively F-Solving on $\mathrm{f}_{1}$ and the newly generated fact, we generate an infinity of solved facts of the form $\left[f\left(\ldots f\left(w_{1}\right) \ldots\right) \triangleright g(h(\ldots h(a) \ldots))\right]$. Intuitively, this happens because our symbolic representation is unable to express that the function $h$ can be nested an unbounded number of times when it occurs under an application of $g$.

The same kind of limitation already exists in the procedure implemented in YAPA [9]. However, our symbolic representation, that manipulates terms that are not necessarily ground and facts with side conditions, allows us to go beyond YAPA. We are able for instance to treat equational theories such as malleable encryption and trapdoor commitment. 


\subsection{Generic method for proving termination}

We provide a generic method for proving termination, which we instantiate in the following section on several examples.

In order to prove that the saturation algorithm terminates, we require that the update function $\oplus$ be uniform: i.e., the same recipe $R^{\prime}$ be used for all useless solved deduction facts that have the same canonical form. Note that the soundness and completeness of the algorithm does not depend on the choice of the recipe $R^{\prime}$ when updating the knowledge base with a useless fact ( $c f$. Definition 5).

Definition 6 (projection). We define the projection of a deduction fact $\mathrm{f}=$ $\left[R \triangleright t \mid X_{1} \triangleright t_{1}, \ldots, X_{n} \triangleright t_{n}\right]$ as $\hat{\mathrm{f}}=\left[t \mid\left\{t_{1}, \ldots, t_{n}\right\}\right]$. We extend the projection to sets of facts $\mathrm{F}$ and define $\hat{\mathrm{F}}=\{\hat{\mathrm{f}} \mid \mathrm{f} \in \mathrm{F}\}$.

We identify projections which are equal up to bijective renaming of variables and we sometimes omit braces for the side conditions.

Proposition 3 (generic termination). The saturation algorithm terminates if $\oplus$ is uniform and there exist some functions $\mathcal{Q}, \mathrm{m}_{\mathrm{f}}, \mathrm{m}_{\mathrm{e}}$ and some wellfounded orders $<_{\mathrm{f}}$ and $<_{\mathrm{e}}$ such that for all frames $\varphi$, and for all $(\mathrm{F}, \mathrm{E})$ such that $\operatorname{Init}(\varphi) \Longrightarrow^{*}(\mathrm{~F}, \mathrm{E})$, we have that:

1. $\{\hat{\mathrm{f}} \mid \mathrm{f} \in \mathrm{F}$ and $\mathrm{f}$ is a solved deduction fact $\} \subseteq \mathcal{Q}(\varphi)$ and $\mathcal{Q}(\varphi)$ is finite;

2. $\mathrm{m}_{\mathrm{f}}\left(\mathrm{f}_{0}\right)<_{\mathrm{f}} \mathrm{m}_{\mathrm{f}}\left(\mathrm{f}_{1}\right)$ where $\mathrm{f}_{0}, \mathrm{f}_{1}$ are defined as in rule F-Solving;

3. $\mathrm{m}_{\mathrm{e}}\left(\mathrm{f}_{0}\right)<_{\mathrm{e}} \mathrm{m}_{\mathrm{e}}\left(\mathrm{f}_{1}\right)$ where $\mathrm{f}_{0}, \mathrm{f}_{1}$ are defined as in rule E-Solving.

\subsection{Applications}

We now give several examples for which the saturation procedure indeed terminates. For each of these theories the definition of the function $\mathcal{Q}$ relies on the following notion of extended subterms.

Definition 7 (extended subterm). Let $t$ be a term; its set of extended subterms $\operatorname{st}_{\mathcal{R}_{\mathcal{E}}}(t)$ (w.r.t. $\left.\mathcal{E}\right)$ is the smallest set such that:

1. $t \in \mathrm{st}_{\mathcal{R}_{\mathcal{E}}}(t)$,

2. $f\left(t_{1}, \ldots, t_{k}\right) \in \mathrm{st}_{\mathcal{R}_{\mathcal{E}}}(t)$ implies $t_{1}, \ldots, t_{k} \in \mathrm{st}_{\mathcal{R}_{\mathcal{E}}}(t)$,

3. $t^{\prime} \in \mathrm{st}_{\mathcal{R}_{\mathcal{E}}}(t)$ and $t^{\prime} \rightarrow \mathcal{R}_{\mathcal{E}} t^{\prime \prime}$ implies $t^{\prime \prime} \in \mathrm{st}_{\mathcal{R}_{\mathcal{E}}}(t)$.

This notation is extended to frames in the usual way.

All the examples in this section rely on the same measures $m_{f}$ and $m_{e}$. Let $\left\{X_{1} \triangleright t_{1}, \ldots, X_{n} \triangleright t_{n}\right\}$ be the set of side conditions of a fact $f$. We define $\mathrm{m}_{\mathrm{f}}(\mathrm{f})=\left(\# \operatorname{var}\left(t_{1}, \ldots, t_{n}\right), \sum_{1<i<n}\left|t_{i}\right|\right)$ and $<_{\mathrm{f}}$ is the lexicographical order on ordered pairs of integers. The measure $m_{e}$ and the order $<_{e}$ are defined in the same way.

We now present the class of subterm convergent equational theories as well as the theories for malleable encryption and trap-door commitment. 
Subterm convergent equational theories. Abadi and Cortier [1] have shown that deduction and static equivalence are decidable for subterm convergent equational theories in polynomial time. We retrieve the same results with our algorithm. An equational theory $\mathcal{E}$ is subterm convergent if $\mathcal{R}_{\mathcal{E}}$ is convergent and for every rule $l \rightarrow r \in \mathcal{R}_{\mathcal{E}}$, we have that either $r$ is a strict subterm of $l$, or $r$ is a ground term in $\mathcal{R}_{\mathcal{E}}$-normal form.

The termination proof for this class relies on the function $\mathcal{Q}$ where $\mathcal{Q}(\varphi)$ is defined as the smallest set that contains

1. $[t \mid \emptyset]$, where $t \in \operatorname{st}_{\mathcal{R}_{\mathcal{E}}}(\varphi)$;

2. $\left[f\left(x_{1}, \ldots, x_{k}\right) \mid x_{1}, \ldots, x_{k}\right]$, where $\operatorname{ar}(f)=k$.

Malleable encryption. We also obtain termination for the equational theory $\mathcal{E}_{m a l}$ described in Example 1. This is a toy example that does not fall in the class studied in [1]. Indeed, this theory is not locally stable: the set of terms in normal form deducible from a frame $\varphi$ cannot always be obtained by applying public contexts over a finite set (called $\operatorname{sat}(\varphi)$ in [1]) of ground terms.

As a witness consider the frame $\varphi_{2}=\nu a, k .\left\{w_{1} \mapsto e n c(b, k\}\right.$ introduced in Example 2. Among the terms that are deducible from $\varphi_{2}$, we have those of the form enc $(t, k)$ where $t$ represents any term deducible from $\varphi_{2}$. From this observation, it is easy to see that $\mathcal{E}_{\text {mal }}$ is not locally stable.

Our procedure does not have this limitation. A prerequisite for termination is that the set of terms in normal form deducible from a frame is exactly the set of terms obtained by nesting in all possible ways a finite set of contexts. The theory $\mathcal{E}_{\text {mal }}$ falls in this class. In particular, for the frame $\varphi_{2}$, our procedure produces the fact $\mathrm{f}_{9}=\left[\operatorname{mal}\left(w_{1}, Y_{2}\right) \triangleright \operatorname{enc}(z, k) \mid Y_{2} \triangleright z\right]$ allowing us to capture all the terms of the form enc $(t, k)$ by the means of a single deduction fact.

The termination proof relies on the function $\mathcal{Q}$ where $\mathcal{Q}(\varphi)$ is defined as the smallest set that contains:

1. $[t \mid \emptyset]$, for every $t \in \operatorname{st}_{\mathcal{R}_{\mathcal{E}}}(\varphi)$;

2. $\left[f\left(x_{1}, x_{2}\right) \mid x_{1}, x_{2}\right]$, where $f \in\{$ enc, dec, mal $\}$;

3. $[\operatorname{enc}(x, t) \mid x]$, if there exists $t^{\prime}$ such that enc $\left(t^{\prime}, t\right) \in \operatorname{st}_{\mathcal{R}_{\mathcal{E}}}(\varphi)$.

Trap-door commitment. The following convergent equational theory $\mathcal{E}_{t d}$ is a model for trap-door commitment:

$$
\begin{array}{ll}
\operatorname{open}(t d(x, y, z), y)=x & t d\left(x_{2}, f\left(x_{1}, y, z, x_{2}\right), z\right)=t d\left(x_{1}, y, z\right) \\
\operatorname{open}\left(t d\left(x_{1}, y, z\right), f\left(x_{1}, y, z, x_{2}\right)\right)=x_{2} & f\left(x_{2}, f\left(x_{1}, y, z, x_{2}\right), z, x_{3}\right)=f\left(x_{1}, y, z, x_{3}\right)
\end{array}
$$

As said in the introduction, we encountered this equational theory when studying electronic voting protocols. The term $t d(m, r, t d)$ models the commitment of the message $m$ under the key $r$ using an additional trap-door $t d$. Such a commitment scheme allows a voter who has performed a commitment to open it in different ways using its trap-door. Hence, trap-door bit commitment $t d(v, r, t d)$ does not bind the voter to the vote $v$. This is useful to ensure privacytype properties in e-voting and in particular receipt-freeness [19]. With such a 
scheme, even if a coercer requires the voter to reveal his commitment, this does not give any useful information to the coercer as the commitment can be viewed as the commitment of any vote (depending on the key that will be used to open it).

For the same reason as $\mathcal{E}_{\text {mal }}$, the theory of trap-door commitment described below cannot be handled by the algorithms described in [1,9]. Our termination proof relies on the function $\mathcal{Q}$ where $\mathcal{Q}(\varphi)$ is the smallest set that contains:

1. $[t \mid \emptyset]$, for every $t \in \operatorname{st}_{\mathcal{R}_{\mathcal{E}}}(\varphi)$;

2. $\left[t d\left(t_{1}, r, t p\right) \mid \emptyset\right]$ such that $f\left(t_{1}, r, t p, t_{2}\right) \in \operatorname{st}_{\mathcal{R}_{\mathcal{E}}}(\varphi)$ for some $t_{2}$;

3. $\left[g\left(x_{1}, \ldots, x_{k}\right) \mid x_{1}, \ldots, x_{k}\right]$, where $g \in\{$ open, $t d, f\}$ and $\operatorname{ar}(g)=k$;

4. $\left[f\left(t_{1}, r, t p, x\right) \mid x\right]$, such that $f\left(t_{1}, r, t p, t_{2}\right) \in \operatorname{st}_{\mathcal{R}_{\mathcal{E}}}(\varphi)$ for some $t_{2}$.

Termination of our procedure is also ensured for theories such as blind signature and addition as defined in [1].

\subsection{Going beyond with fair strategies}

In [1] decidability is also shown for an equational theory modeling homomorphic encryption. For our procedure to terminate on this theory we use a particular saturation strategy.

Homomorphic encryption. We consider the theory $\mathcal{E}_{\text {hom }}$ of homomorphic encryption that has been studied in $[1,9]$.

$$
\begin{gathered}
f \operatorname{st}(\operatorname{pair}(x, y))=x \quad \operatorname{snd}(\operatorname{pair}(x, y))=y \quad \operatorname{dec}(\operatorname{enc}(x, y), y)=x \\
\operatorname{enc}(\operatorname{pair}(x, y), z)=\operatorname{pair}(\operatorname{enc}(x, z), \operatorname{enc}(y, z)) \\
\operatorname{dec}(\operatorname{pair}(x, y), z)=\operatorname{pair}(\operatorname{dec}(x, z), \operatorname{dec}(y, z))
\end{gathered}
$$

In general, our algorithm does not terminate under this equational theory. Consider for instance the frame $\phi=\nu a, b .\left\{w_{1} \mapsto \operatorname{pair}(a, b)\right\}$. We have that:

$$
\operatorname{Init}(\varphi) \supseteq\left\{\left[w_{1} \triangleright \operatorname{pair}(a, b)\right],[\operatorname{enc}(X, Y) \triangleright \operatorname{enc}(x, y) \mid X \triangleright x, Y \triangleright y]\right\} .
$$

As in Example 7 we can obtain an unbounded number of solved facts whose projections are of the form:

$$
\left[\operatorname{pair}\left(\operatorname{enc}\left(\ldots \operatorname{enc}\left(a, z_{1}\right) \ldots, z_{n}\right), \operatorname{enc}\left(\ldots \operatorname{enc}\left(b, z_{1}\right) \ldots, z_{n}\right)\right) \mid z_{1}, \ldots, z_{n}\right] .
$$

However, we can guarantee termination by using a fair saturation strategy. We say that a saturation strategy is fair if whenever a rule instance is enabled it will eventually be taken.

Indeed in the above example using a fair strategy we will eventually add the facts $\left[f s t\left(w_{1}\right) \triangleright a\right]$ and $\left[\operatorname{snd}\left(w_{1}\right) \triangleright b\right]$. Now the "problematic" facts described above become useless and are not added to the knowledge base anymore. One may note that a fair strategy does not guarantee termination in Example 7 (intuitively, because the function $g$ is one-way and $a$ is not deducible in that example).

The proof of termination will as for the previous theories define functions $\mathcal{Q}$, $\mathrm{m}_{\mathrm{f}}$ and $\mathrm{m}_{\mathrm{e}}$. The main argument of the proof is the observation that due to fairness only a finite number of solved fact not in $\mathcal{Q}(\varphi)$ can be added. 


\section{Implementation}

A $\mathrm{C}++$ implementation of the procedures described in this paper is provided in the KISs (Knowledge In Security protocols) tool [11]. The tool implements a uniform $\oplus$ and contains several optimizations. First, as the order of solving side conditions is not important, we always solve the first unsolved side condition rather than considering all the combinations. We also use DAG representation of terms and specialized F-Solving and E-Solving rules for solving ground side conditions. Indeed, by checking whether the side condition is generated or not we know whether solving it will eventually produce a solved fact. Checking generation takes only polynomial time. This makes the procedure terminate in polynomial time for subterm convergent equational theories, and the theories $\mathcal{E}_{\text {blind }}, \mathcal{E}_{\text {mal }}$ and $\mathcal{E}_{t d}$.

The performance of the tool is comparable to the YAPA tool $[8,9]$ and on most examples the tool terminates in less than a second. In [9] a family of contrived examples is presented to diminish the performance of YAPA, exploiting the fact that YAPA does not implement DAG representations of terms and recipes, as opposed to KISs. As expected, KISs indeed performs better on these examples.

Regarding termination, our procedure terminates on all examples of equational theories presented in [9]. In addition, our tool terminates on the theories $\mathcal{E}_{m a l}$ and $\mathcal{E}_{t d}$ whereas YAPA does not. In [9] a class of equational theories for which YAPA terminates is identified and it is not known whether our procedure terminates. YAPA may also terminate on examples outside this class. Hence the question whether termination of our procedures encompasses termination of YAPA is still open.

\section{Conclusion}

We have proposed a procedure for deduction and for static equivalence for convergent equational theories. Our procedure terminates for a wide range of equational theories. In particular, we obtain a new decidability result for the theory of trapdoor commitment.

As future work, we indent to extend our approach in order to handle associative commutative operators (like xor) and the active case of the two problems.

\section{References}

1. M. Abadi and V. Cortier. Deciding knowledge in security protocols under equational theories. Theoretical Computer Science, 387(1-2):2-32, 2006.

2. M. Abadi and C. Fournet. Mobile values, new names, and secure communication. In Proc. 28th ACM Symp. on Principles of Programming Languages, 2001.

3. S. Anantharaman, P. Narendran, and M. Rusinowitch. Intruders with caps. In Proc. 18th International Conference on Term Rewriting and Applications (RTA'07), volume 4533 of LNCS. Springer, 2007. 
4. A. Armando et al. The AVISPA Tool for the automated validation of internet security protocols and applications. In Proc. 17th Int. Conference on Computer Aided Verification (CAV'05), volume 3576 of LNCS, pages 281-285. Springer, 2005.

5. M. Backes, C. Hritcu, and M. Maffei. Automated verification of remote electronic voting protocols in the applied pi-calculus. In Proc. 21st IEEE Computer Security Foundations Symposium (CSF'08), 2008.

6. M. Backes, M. Maffei, and D. Unruh. Zero-knowledge in the applied pi-calculus and automated verification of the direct anonymous attestation protocol. In IEEE Symposium on Security and Privacy (SEP'O8). IEEE Comp. Soc. Press, 2008.

7. M. Baudet. Deciding security of protocols against off-line guessing attacks. In 12th ACM Conference on Computer and Communications Security (CCS'05), 2005.

8. M. Baudet. YAPA (Yet Another Protocol Analyzer), 2008. http://www.lsv.enscachan.fr/ ${ }^{\sim}$ baudet/yapa/index.html.

9. M. Baudet, V. Cortier, and S. Delaune. YAPA: A generic tool for computing intruder knowledge. In R. Treinen, editor, Proceedings of the 20th International Conference on Rewriting Techniques and Applications (RTA'09), Lecture Notes in Computer Science, Brasília, Brazil, June-July 2009. Springer. To appear.

10. Y. Chevalier. Résolution de problèmes d'accessibilité pour la compilation et la validation de protocoles cryptographiques. PhD thesis, Univ. Henri Poincaré, 2003.

11. Ş. Ciobâcă. KISs, 2009. http://www.lsv.ens-cachan.fr/ ciobaca/kiss.

12. Ş. Ciobâcă, S. Delaune, and S. Kremer. Computing knowledge in security protocol under convergent equational theories. Research Report LSV-09-05, Laboratoire Spécification et Vérification, ENS Cachan, France, Mar. 2009. 42 pages.

13. R. Corin, J. Doumen, and S. Etalle. Analysing password protocol security against off-line dictionary attacks. In Proc. 2nd International Workshop on Security Issues with Petri Nets and other Computational Models (WISP'04), ENTCS, 2004.

14. V. Cortier and S. Delaune. Deciding knowledge in security protocols for monoidal equational theories. In Proc. 14th Int. Conference on Logic for Programming, Artificial Intelligence, and Reasoning (LPAR'07), LNAI. Springer, 2007.

15. V. Cortier, S. Delaune, and P. Lafourcade. A survey of algebraic properties used in cryptographic protocols. Journal of Computer Security, 14(1):1-43, 2006.

16. S. Delaune, S. Kremer, and M. D. Ryan. Verifying privacy-type properties of electronic voting protocols. Journal of Computer Security, 2008. To appear.

17. P. Lafourcade, D. Lugiez, and R. Treinen. Intruder deduction for the equational theory of Abelian groups with distributive encryption. Information and Computation, 205(4):581-623, 2007.

18. J. Millen and V. Shmatikov. Constraint solving for bounded-process cryptographic protocol analysis. In Proc. 8th ACM Conference on Computer and Communications Security (CCS'01), 2001.

19. T. Okamoto. Receipt-free electronic voting schemes for large scale elections. In Proc. 5th Int. Security Protocols Workshop, volume 1361. Springer, 1997.

20. M. Rusinowitch and M. Turuani. Protocol insecurity with finite number of sessions and composed keys is NP-complete. Theoretical Computer Science, 299:451-475, 2003. 